\title{
Regression of Retinopathy by Squalamine in a Mouse Model
}

\author{
ROSEMARY D. HIGGINS, YUN YAN, YIXUN GENG, MICHAEL ZASLOFF, AND \\ JON I. WILLIAMS \\ Department of Pediatrics, Division of Neonatology [R.D.H., Y.Y., Y.G.], Office of the Dean of \\ Translational Research [M.Z.], Georgetown University, Washington, DC, 20007, U.S.A., and Genaera \\ Corporation, Plymouth Meeting, Pennsylvania 19462, U.S.A. [J.I.W.]
}

\begin{abstract}
The goal of this study was to determine whether an antiangiogenic agent, squalamine, given late during the evolution of oxygen-induced retinopathy (OIR) in the mouse, could improve retinal neovascularization. OIR was induced in neonatal C57BL6 mice and the neonates were treated s.c. with squalamine doses begun at various times after OIR induction. A system of retinal whole mounts and assessment of neovascular nuclei extending beyond the inner limiting membrane from animals reared under room air or OIR conditions and killed periodically from d 12 to 21 were used to assess retinopathy in squalamine-treated and untreated animals. OIR evolved after $75 \%$ oxygen exposure in neonatal mice with florid retinal neovascularization developing by d 14 . Squalamine (single dose, $25 \mathrm{mg} / \mathrm{kg}$ s.c.) given on $\mathrm{d} 15$ or 16 , but not d 17 , substantially improved retinal neovascularization in the mouse model of OIR. There was improvement seen
\end{abstract}

ABSTRACT

in the degree of blood vessel tuft formation, blood vessel tortuosity, and central vasoconstriction with squalamine treatment at d 15 or 16 . Single-dose squalamine at d 12 was effective at reducing subsequent development of retinal neovascularization at doses as low as $1 \mathrm{mg} / \mathrm{kg}$. Squalamine is a very active inhibitor of OIR in mouse neonates at doses as low as $1 \mathrm{mg} / \mathrm{kg}$ given once. Further, squalamine given late in the course of OIR improves retinopathy by inducing regression of retinal neovessels and abrogating invasion of new vessels beyond the inner-limiting membrane of the retina. (Pediatr Res 56: 144-149, 2004)
Abbreviations
OIR, oxygen-induced retinopathy
VEGF, vascular endothelial growth factor

Retinopathy is a significant and sight-threatening ophthalmologic condition. Current modalities for treatment of various forms of retinopathy involve surgical interventions such as laser ablative treatment and/or cryotherapy late in the disease (i.e. when retinal detachment or blindness are likely to occur). Certainly therapy aimed at inhibition or reversal of retinal neovascularization could be used clinically before the current surgical treatments for retinopathy. There are not preventive or early intervention modalities of treatment currently available for retinopathy of prematurity or diabetic retinopathy. If therapy were made available early in the course of retinal neovascularization, outcome could be greatly impacted.

Squalamine is an aminosterol antibiotic (1) that has been shown to improve retinal neovascularization (2). It also has

Received April 1, 2003; accepted October 23, 2003.

Correspondence: Rosemary D. Higgins, M.D., Pregnancy and Perinatology Branch, Center for Developmental Biology and Perinatal Medicine, National Institute of Child Health and Human Development, National Institutes of Health, 6100 Executive Blvd., Room 4B03B, MSC 7510, Bethesda, MD 20892, U.S.A.; e-mail: higginsr@mail.nih.gov

Supported by Juvenile Diabetes Foundation International Research Grant 1-2000-334 and the Genaera Corporation.

DOI: 10.1203/01.PDR.0000128977.55799.34 been shown to inhibit angiogenesis in various tumor models (some with combination therapy), including rat 9L glioma (3), mammary carcinoma (4), Lewis lung carcinoma (4), and ovarian cancer (5). The mechanism of action of squalamine for inhibition of angiogenesis is proposed to be inhibition of VEGF-mediated endothelial cell proliferation (3). More specifically, squalamine has been shown to block VEGF-induced activation of mitogen-activated protein kinase in human vascular endothelial cells (5).

Our laboratory recently demonstrated inhibition of retinal neovascularization by squalamine in a mouse model of OIR when given systemically for 1 and $5 \mathrm{~d}$ (2). Systemic squalamine also has been shown to inhibit iris neovascularization in cynomolgus monkeys at late stages of neovessel development, suggesting that the relative timing of squalamine application during neovessel formation in the eye may be important (6). We propose that therapy with squalamine could potentially be used throughout the evolution of the course of retinopathy.

The goal of this study was to determine a minimal effective dose of squalamine for use in a multidosing regime and to determine how late in the course of OIR squalamine 
Table 1. Retinal scoring system

\begin{tabular}{|c|c|c|c|c|c|}
\hline \multirow[b]{2}{*}{ Categories } & \multicolumn{5}{|c|}{ Scores } \\
\hline & 0 & 1 & 2 & 3 & 4 \\
\hline Blood vessel growth & Complete & Incomplete outer third & $\begin{array}{l}\text { Incomplete middle } \\
\text { third }\end{array}$ & $\begin{array}{l}\text { Incomplete inner } \\
\text { third }\end{array}$ & - \\
\hline Blood vessel tufts & None & Few, scattered $(<3 \mathrm{~h})$ & $3-5 \mathrm{~h}$ & $6-8 \mathrm{~h}$ & $9-12 \mathrm{~h}$ \\
\hline $\begin{array}{l}\text { Extraretinal } \\
\text { neovascularization }\end{array}$ & None & Mild $(<3 \mathrm{~h})$ & Moderate $(3-6$ h) & Severe $(>6 \mathrm{~h})$ & - \\
\hline $\begin{array}{l}\text { Central } \\
\text { vasoconstriction }\end{array}$ & None & $\begin{array}{l}\text { Mild, early zone } 1 \text { (inner } \\
50 \% \text { of zone } 1 \text { ) }\end{array}$ & $\begin{array}{l}\text { Moderate. Throughout } \\
\text { zone } 1\end{array}$ & $\begin{array}{l}\text { Severe (extending } \\
\text { in zone } 2 \text { ) }\end{array}$ & - \\
\hline Retinal hemorrhage & Absent & Present & - & - & \\
\hline Blood vessel tortuosity & None & Mild (<3 h) & Moderate (3-6 h) & Severe $(>6 \mathrm{~h})$ & - \\
\hline
\end{tabular}

Fluorescein-angiography-performed flat retinal mounts were assessed for severity of retinopathy with this scoring system. h, clock hours of pathology. (Reprinted with permission from Higgins RD, et al. Current Eye Research 1999; 18: 22. OSwets \& Zeitlinger Publishers).

could be used to provide a beneficial effect for retinal neovascularization.

\section{METHODS}

Animal model. C57BL6 mice were obtained from Taconic Farms (Germantown, NY, U.S.A.). Infant mice were placed in an incubator with their nursing mother with $75 \%$ oxygen starting at postnatal d 7 (P7) through P12 as previously described (7) and as used in our laboratory (2). The oxygen was delivered at $75 \% \pm 2 \%$, measured with an oxygen analyzer (Hudson Ventronics, Temecula, CA, U.S.A.), and checked at least twice daily. On P12, animals were returned to room air. Animals were subsequently killed at various time points up to P21 using a lethal dose of sodium pentobarbital $(120 \mathrm{mg} / \mathrm{kg})$. This protocol was reviewed and approved by the Georgetown University Animal Care and Use Committee and adhered to the Association for Research in Vision and Ophthalmology Statement for the Use of Animals in Ophthalmic and Vision Research.

Time course of retinopathy. To determine the daily changes occurring in the mouse model of OIR, animals were killed from d 12 through d 17 after exposure to hyperoxic conditions. Retinal whole mounts and retinal sections were prepared to determine the degree of retinopathy on the given days.

Squalamine administration. Squalamine (provided by Genaera Corporation, Plymouth Meeting, PA, U.S.A.) was administered s.c. in the nape of the neck. Doses from 1 to 25 $\mathrm{mg} / \mathrm{kg}$ in a single daily injection at P12 were initially performed. Squalamine $(25 \mathrm{mg} / \mathrm{kg})$ was given in a single daily injection at P15, P16, and P17 in subsequent experiments to investigate the hypothesis that squalamine can halt or inhibit retinal neovascularization.

Retinal perfusion and scoring of retinal whole mounts. Fluorescein-conjugated dextran perfusion of the retinal vessels was performed as previously described (8) using highmolecular-weight fluorescein conjugated dextran with $4 \%$ paraformaldehyde in PBS. Briefly, animals were given a lethal dose of sodium pentobarbital and when deep anesthesia was obtained, a median sternotomy was performed, and the left ventricle was perfused with $1 \mathrm{~mL}$ of a $50 \mathrm{mg} / \mathrm{mL}$ solution of fluorescein-conjugated dextran. The eyes were enucleated, placed in 4\% paraformaldehyde in PBS, and fixed for 3-24 h. The retinas were dissected under light microscopy and flat mounts were obtained. Retinas were scored in a masked fashion using a previously validated retinopathy scoring system as shown in Table 1 (9).

$P A S$ staining of retinal sections and scoring of extraretinal neovessels. Animals were given a lethal dose of sodium pen-

A

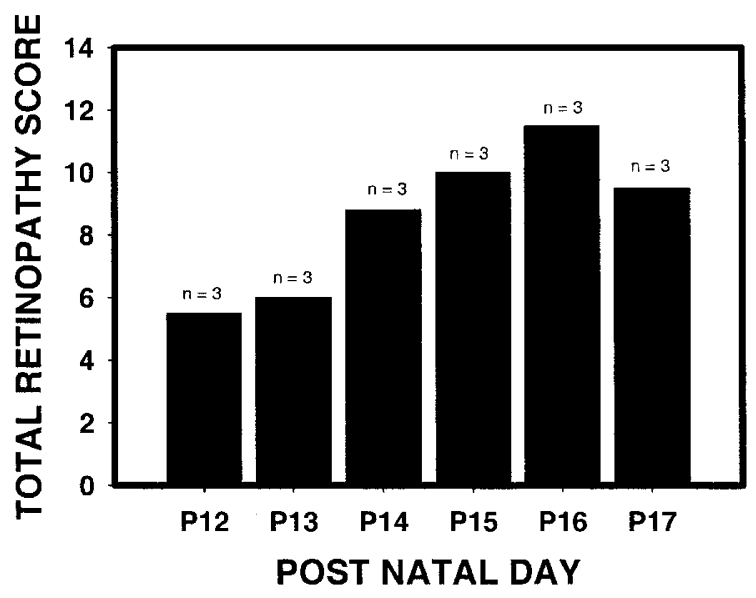

B

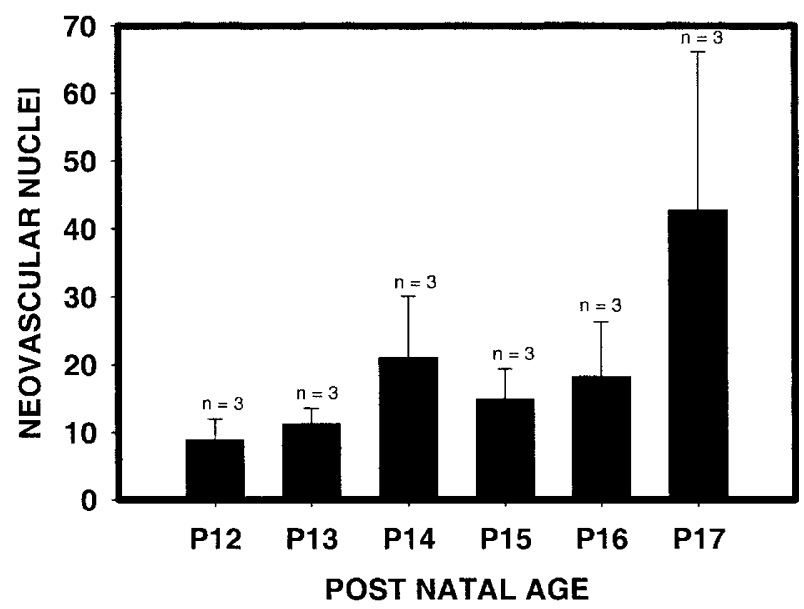

Figure 1. Time course of development of retinopathy score in oxygenexposed animals. $(A)$ Total retinopathy score (median $\pm 75^{\text {th }}$ quartile) as assessed by evaluation of the retinal whole-mount preparations. There is a steady rise in retinopathy score until d 16 in oxygen-treated animals. $(B)$ Time course for neovascular nuclei extending beyond the inner-limiting membrane in oxygen exposed animals. Age in postnatal days is shown on the $x$ axis and neovascular nuclei (mean $\pm \mathrm{SD}$ ) are depicted on the $y$ axis. 
Table 2. Retinopathy subscores [median $\left(25^{\text {th }}, 75^{\text {th }}\right.$ quartiles $)$ ] for time course of oxygen-induced retinopathy

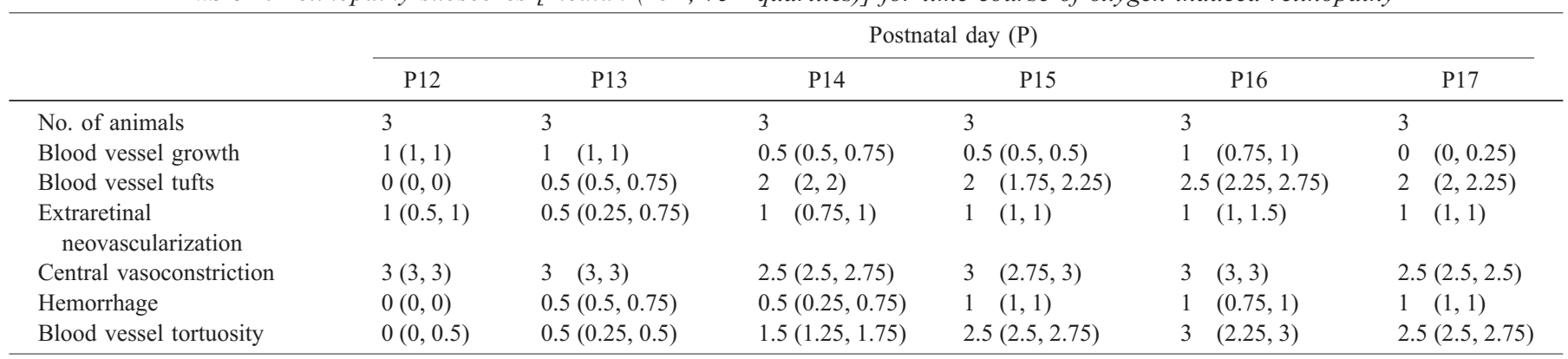

tobarbital and, when deep anesthesia was obtained, underwent a median sternotomy and left ventricular perfusion with $4 \%$ paraformaldehyde in PBS. Eyes were removed, placed in optimum cutting temperature compound (Sakura Finetek, Torrance, CA, U.S.A.), and frozen immediately to $-70^{\circ} \mathrm{C}$. Serial sections $(7-9 \mu \mathrm{m})$ were performed as previously described using a cryostat. Tissue sections were stained with periodic acid-Schiff (PAS) stain and counterstained with hematoxylin as previously described (7) and used in our laboratory $(2,9)$. Multiple sections were scored by counting the number of nuclei extending beyond the inner limiting membrane into the vitreous in a masked fashion. A minimum of six sections at least $50 \mu \mathrm{m}$ apart over a minimum distance of $300 \mu \mathrm{m}$ were counted and averaged for each eye.
Statistical analyses. ANOVA using the Kruskal-Wallis test was performed to detect difference trends between all treatment groups. The Mann-Whitney test was used to compare the total retinopathy scores and retinopathy subscores between individual groups. ANOVA was performed to detect differences between all treatment groups. A $t$ test assuming unequal variance was performed to compare the number of extraretinal nuclei in the retinal sections. Statistical significance was defined as $p<$ 0.05 .

\section{RESULTS}

Time course of retinopathy. Retinopathy as assessed by a previously validated retinopathy scoring system in fluorescein-

A

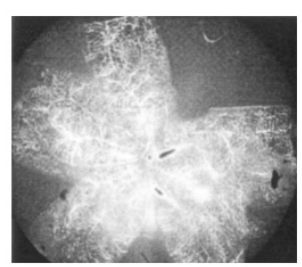

\section{$15 \mathrm{~mm}-$}

B

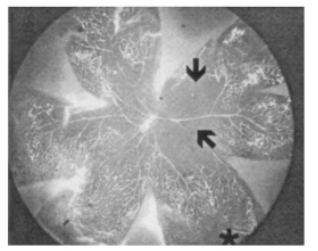

$\mathrm{C}$

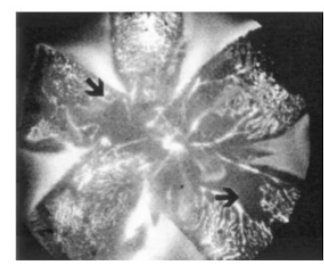

D

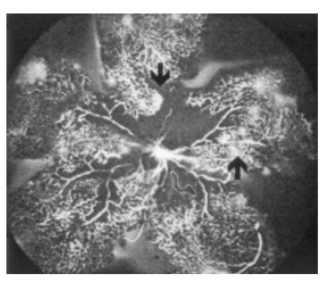

E

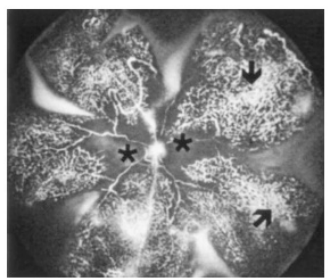

F

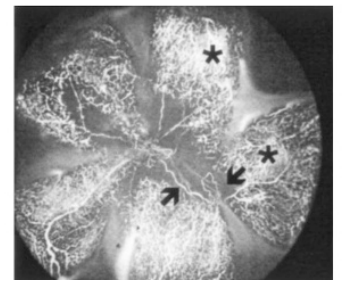

G

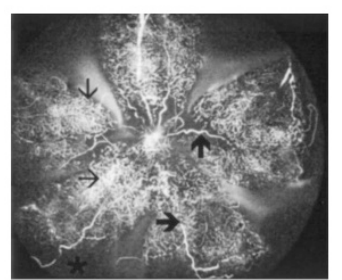

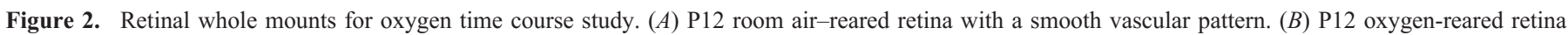

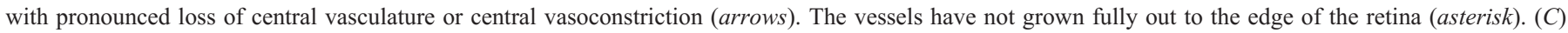

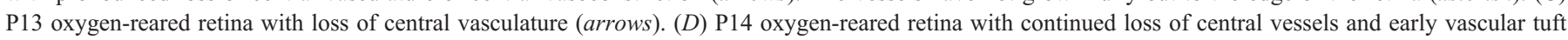

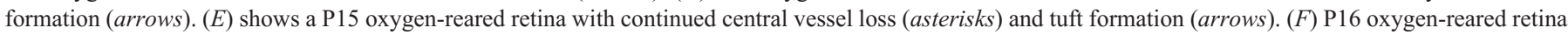

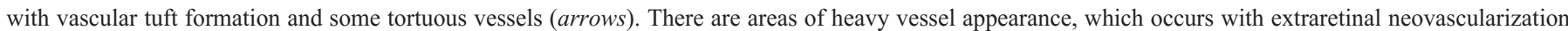

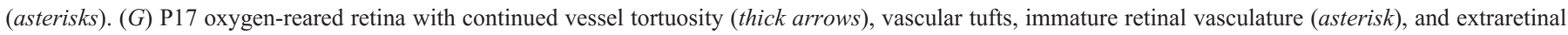
neovascularization (thin arrows). 


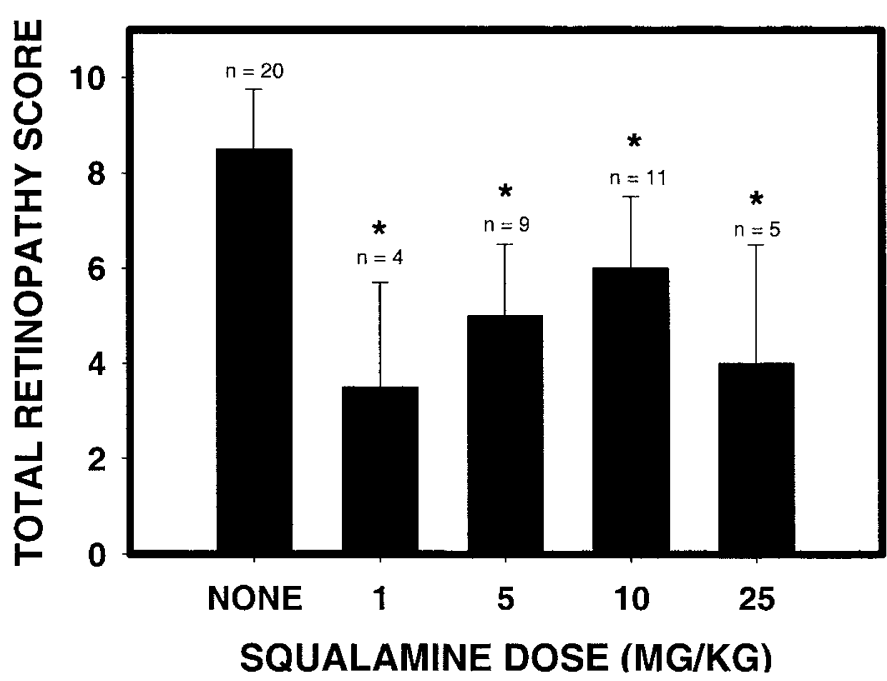

Figure 3. Squalamine dose-response study. A single dose of squalamine was administered s.c. on $\mathrm{d} 12$. The doses of squalamine are depicted on the $x$ axis and total retinopathy scores (median $\pm 75^{\text {th }}$ quartile) are on the $y$ axis. *Denotes $p<0.05$ when compared with no drug group. There were no statistical differences between any of the squalamine doses by ANOVA.

conjugated dextran perfused retinal whole mount preparations ( $n=3$ /group) evolved over the first 2-3 d after exposure to $75 \%$ oxygen, as shown in Figure $1 \mathrm{~A}$. Retinopathy subscores are shown in Table 2 and whole mounts are depicted in Figure 2. At P12, retinal whole mounts from animals exposed to oxygen characteristically showed marked loss of central blood vessels with little presence of blood vessel tufts. However, blood vessel tufts were present between the area of central vessel loss and the growing neovascular front by d 14 and increased thereafter. Retinal hemorrhages were first seen on P13-14 and persisted through P17. The number of extraretinal nuclei extending beyond the inner limiting membrane ( $n=3 /$ group) increased over time as shown in Figure $1 B$, as did blood vessel tufts and extraretinal neovascularization (Table 2).

Effect of single-dose squalamine at P12. Squalamine was administered as a single dose on P12 and retinal neovascularization was evaluated from P17 to P20. A dose of $25 \mathrm{mg} / \mathrm{kg}$ of squalamine given subcutaneously was used based on prior experience (2). However, doses as low as $1 \mathrm{mg} / \mathrm{kg}$ squalamine were found to be equally effective in reducing retinal neovascularization in this model of retinopathy ( $p=$ NS by ANOVA), as shown in Figure 3.

Temporal effects of squalamine on retinal neovascularization. Single-dose squalamine $(25 \mathrm{mg} / \mathrm{kg})$ improved retinal neovascularization when administered at P15 $(n=12)$ or P16 $(n=$ 5 ) and evaluated at P19-21 relative to oxygen-reared animals ( $n$ $=22$ ) as shown in Figures 4 and $5 A$. Total retinopathy scores were statistically improved with a single squalamine dose on P15 or $\mathrm{P} 16$, a time when retinal neovascularization has already reached maximal levels in oxygen-reared animals (Table 3). By contrast, at $\mathrm{d} 17(n=8)$, there was no difference in retinopathy between untreated or squalamine-treated oxygen-reared animals; retinopathy subscore data confirming this observation are summarized in Table 3. Notably, there was significant improvement in P15 animals treated with squalamine in blood vessel tuft formation, extraretinal neovascularization, central vasoconstriction, hemorrhage, and the extent of blood vessel tortuosity as shown in

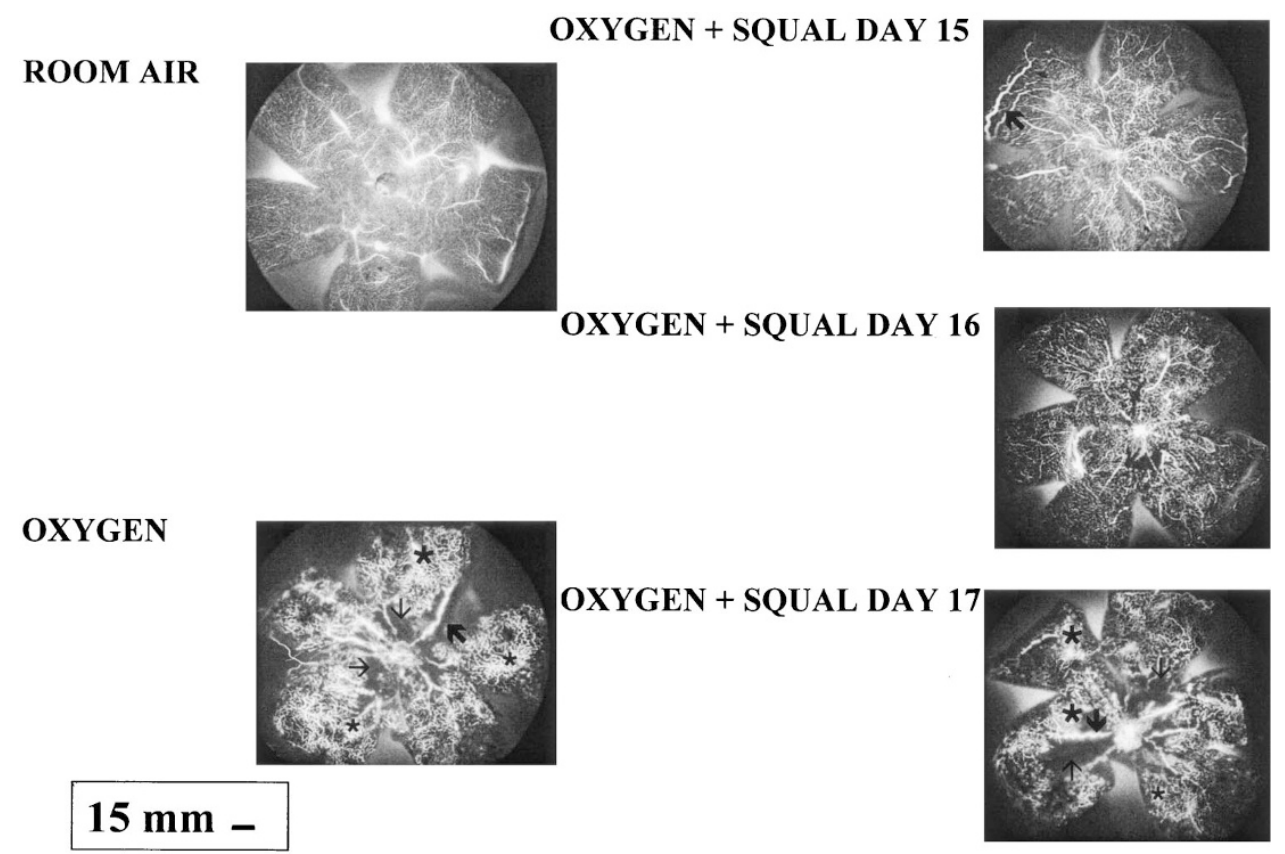

Figure 4. Retinal whole mounts from late squalamine experiments. Room air and oxygen and squalamine treatment at $\mathrm{d} 15,16,17$ are depicted in the photograph. The room air-reared retina has a smooth vascular pattern. The oxygen retina (taken at d 17) has loss of central blood vessels (thin arrows), tortuous blood vessels (thick arrow), vascular tufts (small asterisks), and extraretinal neovascularization (large asterisk). The squalamine and oxygen-treated retinas at d 15 and 16 have very little vascular pathology. There are some tortuous vessels on the retinal whole mount on d 15 (arrow). Likewise, the d 16 squalamine and oxygen-treated retina has less vascular pathology than the oxygen treated retina. At d 17, the squalamine and oxygen whole mount has significant pathology, including loss of central vessels (thin arrows), tortuous vessels (thick arrow), blood vessel tufts (small asterisk), and extraretinal neovascularization (large asterisks). 
A
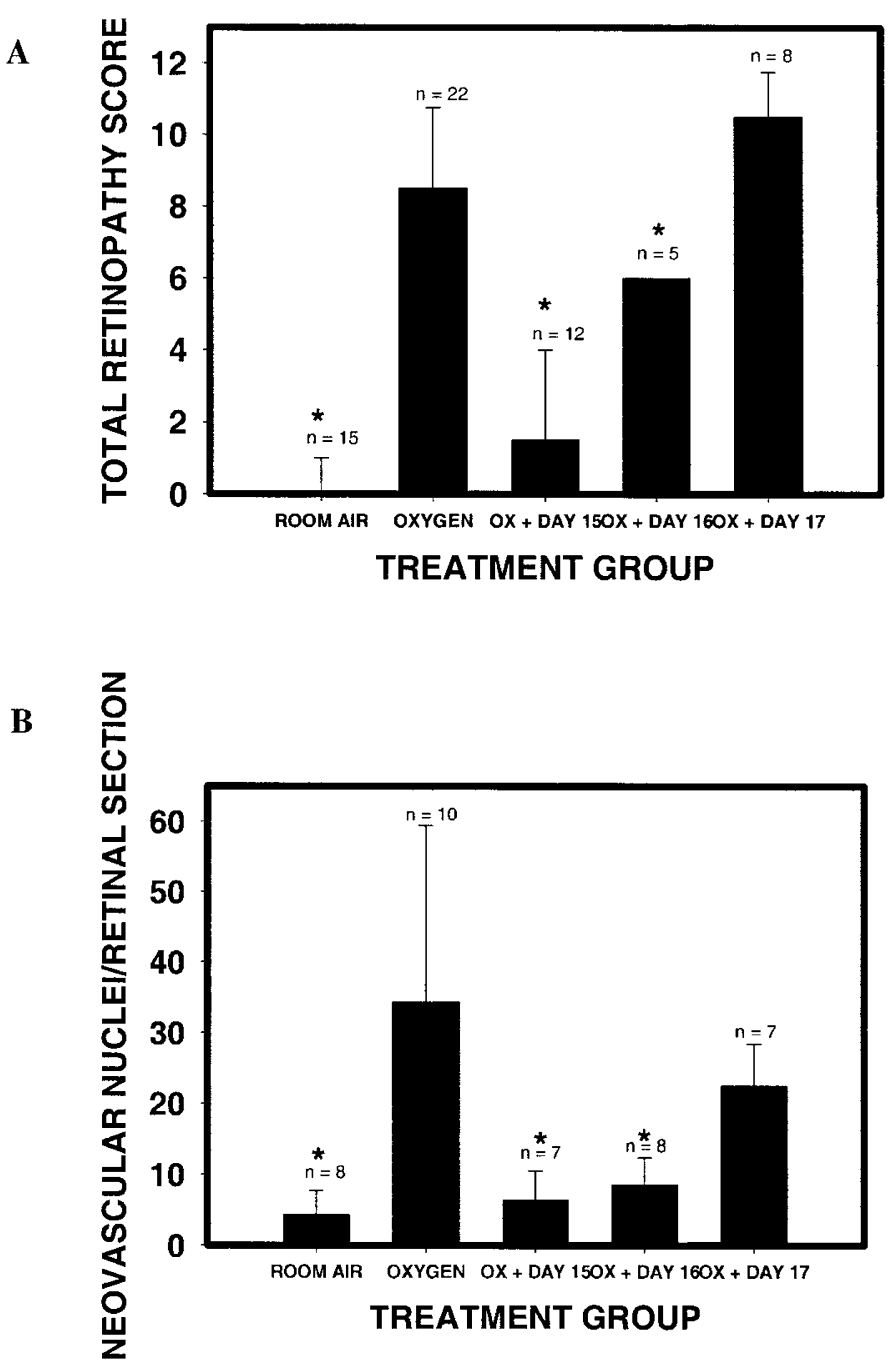

Figure 5. Effect of late squalamine treatment on retinopathy. Squalamine treatment consisted of a single injection of $25 \mathrm{mg} / \mathrm{kg}$ s.c. on the designated day. Treatment groups (room air, oxygen, oxygen plus squalamine at $d 15$, oxygen plus squalamine at $\mathrm{d} 16$, and oxygen plus squalamine at $\mathrm{d} 17$ ) are shown on the $x$ axis. (A) Total retinopathy scores (median $\pm 75^{\text {th }}$ quartile) on the $y$ axis. $(B)$ Neovascular nuclei extending beyond the inner-limiting membrane (mean \pm $\mathrm{SD})$ on the $y$ axis. ${ }^{*} p<0.05$ when compared with oxygen-exposed group.

Figure 4. When animals were treated on P16, they had delayed blood vessel growth, but there was still significant improvement in blood vessel tufts, extraretinal neovascularization, central vasoconstriction, and blood vessel tortuosity as shown in Figure 4.

To corroborate the retinal whole mount data, retinal sections were performed to assess the number of neovascular nuclei extending beyond the inner limiting membrane into the vitreous. Figure $5 B$ shows that animals exposed to hyperoxia and treated with squalamine on P15 or P16 had significantly fewer extraretinal neovascular nuclei than oxygen-reared animals. Data from Figure $1 B$ for the number of neovascular nuclei extending beyond the inner-limiting membrane at P15 in oxygen-reared animals suggests that animals treated on P15 with squalamine should have higher numbers of neovascular nuclei than room air-reared control animals if squalamine simply stops the development of extraretinal neovascular proliferation. This does not occur. Rather, there is significant suppression in the number of extraretinal neovascular nuclei for animals injected with squalamine on P15 and P16 relative to oxygenreared animals. Consequently, these data provide evidence that squalamine causes an apparent true regression of retinal neovascularization when delivered in this model at a time of maximal OIR.

\section{DISCUSSION}

The occurrence of retinopathy or extraretinal neovascular proliferation in the neonatal mouse model of OIR occurs within a few days after removal of the mice from a $75 \%$ oxygen environment, i.e. from d 14 onward. Previous investigators have shown biochemical changes correlating with angiogenesis at this time, but there has been limited information on the evolution of retinopathy with time in this model. Retinal whole mount data and data on the frequency of neovascular nuclei on retinal sections have been obtained on a daily basis from P12 to P17 in the present study to firmly establish a time line for the course of OIR in juvenile mice. Central vasoconstriction or loss of blood vessels is the most marked change at d 12 upon removal of the animals from $75 \%$ oxygen as previously described (7). Blood vessel tufts, extraretinal neovascularization, and blood vessel tortuosity develop gradually from d 12 through d 17 with differing time scales and maximal retinopathy in aggregate appears to occur by P15-P16.

Animals treated with squalamine after retinopathy has maximally occurred (i.e. P15 and P16) show a significant improvement in total retinopathy score and also an improvement in an independent marker of angiogenesis, the number of neovascular nuclei extending beyond the inner limiting membrane. Notably, this improvement does not appear to be just a retardation of the angiogenic process. Animals treated with squalamine on P15 or P16 show less total retinopathy score and less neovascular nuclei than oxygen-reared animals killed at P15 or P16, thus providing evidence for a possible functional role for squalamine in an active process leading to the regression of retinopathy. However, this process is time dependent, as squalamine given at P17 does not have a protective or inhibitory effect on retinopathy.

Squalamine given at d $12(2)$ as a single dose and for $5 \mathrm{~d}$ improves retinopathy in the mouse model. These animals had slightly more central vasoconstriction than the animals treated at d 15 and d 16. After removal from hyperoxia and before squalamine therapy there is an increase in "healing" and, thus, delaying treatment and allowing a natural "wave of healing" may explain the minor differences observed in the two different dosing schemes (i.e. immediate squalamine versus later squalamine). The mechanism of action for squalamine in interfering with ocular vasculopathy may focus on the inhibition of VEGF-dependent events by squalamine inasmuch as squalamine is known to inhibit VEGF stimulation of endothelial cells $(3,5)$. Blocking mitogen-stimulated proliferation and migration of endothelial cells is key to altering or inhibiting retinal neovascularization. As a result, we speculate that squalamine could halt VEGF-stimulated angiogenesis in the retina when administered on d 15 or 16. Alternatively, squalamine may block angiogenesis downstream from VEGF 
Table 3. Retinal subscores [median $\left(25^{\text {th }}, 75^{\text {th }}\right.$ quartile $)$ ] for animals treated with oxygen and late squalamine

\begin{tabular}{|c|c|c|c|c|c|}
\hline Treatment & Room air & Oxygen & Squalamine day 15 & Squalamine day 16 & Squalamine day 17 \\
\hline No. of animals & 8 & 22 & 12 & 5 & 8 \\
\hline Blood vessel growth & $0(0,1)$ & $0 \quad(0,0)$ & $0(0,0)$ & $0.5(0.5,0.9)^{* *}$ & $1.25(1,1.6)^{* *}$ \\
\hline Blood vessel tufts & $0(0,0)$ & $2.5(2,3)^{*}$ & $0(0,0.25)^{* *}$ & $1.25(0.6,1.5)^{* *}$ & $2.5 \quad(2,2.6)$ \\
\hline Central vasoconstriction & $0(0,0)$ & $2(1,2)^{*}$ & $0(0,1)^{* *}$ & $(0.6,1)^{* *}$ & $(1.9,2.6)$ \\
\hline Hemorrhage & $0(0,0)$ & $1 \quad(1,1)^{*}$ & $0(0,0.25)^{* *}$ & $1 \quad(1,1)$ & $1 \quad(1,1)$ \\
\hline
\end{tabular}

$* p<0.05$ compared with room air.

** $p<0.05$ compared with oxygen.

activation of endothelial cells participating in ocular angiogenesis, as evidenced by recent findings of Li et al. (5) showing that squalamine blocks VEGF-induced activation of mitogenactivated protein kinase and cell proliferation in human vascular endothelial cells. Squalamine may also inhibit other factors important in angiogenesis or could potentially stimulate mediators that counter the neovascularization.

The differences observed in the administration of squalamine from d 15 to 17 may be explained on the basis of increased drug clearance in the animals. The half-life of squalamine is approximately $1 \mathrm{~h}$ and terminal elimination occurs after $7.2 \mathrm{~h}$ in humans (10). The time course of retinopathy may be such that a critical window of treatment is over at $\mathrm{d} 17$. The neovascularization may be maximal and beginning to arrest on its own as opposed to being modified by squalamine.

One must be concerned about long-term and systemic effects of angiogenesis inhibition when considering that squalamine may have therapeutic value. For instance, in premature infants with retinopathy of prematurity, the target patient population whom the murine OIR model is intended to mimic, there would be concern regarding systemic angiogenesis inhibition in developing organs such as brain. In adult patients such as those with diabetes mellitus, systemic angiogenesis inhibition may complicate co-morbidities such as coronary artery disease and cutaneous ulcerations. An intermittent or short course dosing regime for retinopathy therefore would be preferred if it were effective. The data presented in this mouse study indicate that squalamine may have the desirable characteristic of being effective after a short dosing course with a substantial reduction in retinal neovascularization.

Squalamine has been studied in advanced cancers in a phase I trial. Hepatotoxicity was the predominant toxicity observed that was reversible upon discontinuation of the drug (10). Transient tumor regression was observed, but was not sustained as this was a single-dose trial. Combination trials involving non-small cell lung cancer and ovarian cancer are underway. Squalamine is currently being evaluated in a phase I/II clinical trial for the treatment of age-related macular degeneration, a disease characterized by the growth of neovascular membranes from the choriocapillaris into the subretinal space. If safety and efficacy of squalamine are shown to be effective in this trial, then perhaps squalamine can be tested in other forms of ocular neovascularization.

Squalamine's ability to block angiogenesis after it has already begun and has achieved maximal neovascularization in the mouse model of OIR is a significant finding, and underscores that squalamine may ultimately prove useful as an agent for treatment of ocular neovascularization.

\section{REFERENCES}

1. Moore KS, Wehrli S, Roder H, Rogers M, Forrest Jr JN, McCrimmon D, Zasloff M 1993 Squalamine: an aminosterol antibiotic from the shark. Proc Natl Acad Sci U S A 90:1354-1358

2. Higgins RD, Sanders RJ, Yan Y, Zasloff M, Williams JI 2000 Squalamine improves retinal neovascularization. Invest Ophthalmol Vis Sci 41:1507-1512

3. Sills AK, Williams JI, Tyler BM, Epstein DS, Sipos EP, Davids JD, McLane MP, Pitchford S, Cheshire K, Gannon FH, Kinney WA, Chao TL, Donowitz M, Laterra J, Brem H 1998 Squalamine inhibits angiogenesis and solid tumor growth in vivo and perturbs embryonic vasculature. Cancer Res 58:2784-2792

4. Teicher BA, Williams JI, Takeuchi H, Ara G, Herbst RS, Buxton D 1998 Potential of the aminosterol squalamine in combination therapy in the rat 13,762 mammary carcinoma and the murine Lewis lung carcinoma. Anticancer Res 18:2567-2573

5. Li D, Williams JI, Peitras RJ 2002 Squalamine and cisplatin block angiogenesis and growth of human ovarian cancer cells with or without HER-2 gene overexpression. Oncogene 21:2805-2814

6. Genaidy M, Kazi AA, Peyman GA, Passos-Machado E, Farahat H, Williams JI, Holroyd K, Blake DA 2002 Effect of squalamine on iris neovascularization in monkeys. Retina 22:772-778

7. Smith LE, Wesolowski E, McLellan A, Kostyk SK, D'Amato R, Sullivan R, D'Amore PA 1994 Oxygen-induced retinopathy in the mouse. Invest Ophthalmol Vis Sci 35:101-111

8. D'Amato R, Wesolowski E, Smith LE 1993 Microscopic visualization of the retina by angiography with high-molecular weight fluorescein-labeled dextrans in the mouse. Microvasc Res 46:135-142

9. Higgins RD, Yu K, Sanders RJ, Nandgaonkar BN, Rotschild T, Rifkin DB 1999 Diltiazem reduces retinal neovascularization in a mouse model of oxygen induced retinopathy. Curr Eye Res 18:20-27

10. Bhargava P, Marshall JL, Dahut W, Rizvi N, Trocky N, Williams JI, Hait H, Song S, Holroyd KJ, Hawkins MJ 2001 A Phase I and pharmacokinetic study of squalamine, a novel antiangiogenic agent, in patients with advanced cancers. Clin Can Res 7:3912-3919 